Research

Open Access

\title{
Exogenous nitric oxide requires an endothelial glycocalyx to prevent postischemic coronary vascular leak in guinea pig hearts
}

\author{
Dirk Bruegger ${ }^{1}$, Markus Rehm¹, Matthias Jacob1, Daniel Chappell1', Mechthild Stoeckelhuber², \\ Ulrich Welsch ${ }^{2}$, Peter Conzen ${ }^{1}$ and Bernhard F Becker ${ }^{3}$
}

\begin{abstract}
${ }^{1}$ Clinic of Anesthesiology, Ludwig-Maximilians-University, Marchioninistrasse 15, 81377 Munich, Germany 2Department of Anatomy, Ludwig-Maximilians-University, Pettenkoferstrasse 11, 80336 Munich, Germany ${ }^{3}$ Department of Physiology, Ludwig-Maximilians-University, Pettenkoferstrasse 12, 80336 Munich, Germany

Corresponding author: Dirk Bruegger, dirk.bruegger@med.uni-muenchen.de
\end{abstract}

Received: 14 Jan 2008 Revisions requested: 15 Feb 2008 Revisions received: 19 Mar 2008 Accepted: 2 Jun 2008 Published: 2 Jun 2008

Critical Care 2008, 12:R73 (doi:10.1186/cc6913)

This article is online at: http://ccforum.com/content/12/3/R73

(C) 2008 Bruegger et al.; licensee BioMed Central Ltd.

This is an open access article distributed under the terms of the Creative Commons Attribution License (http://creativecommons.org/licenses/by/2.0), which permits unrestricted use, distribution, and reproduction in any medium, provided the original work is properly cited.

\begin{abstract}
Introduction Postischemic injury to the coronary vascular endothelium, in particular to the endothelial glycocalyx, may provoke fluid extravasation. Shedding of the glycocalyx is triggered by redox stress encountered during reperfusion and should be alleviated by the radical scavenger nitric oxide (NO). The objective of this study was to investigate the effect of exogenous administration of NO during reperfusion on both coronary endothelial glycocalyx and vascular integrity.

Methods Isolated guinea pig hearts were subjected to 15 minutes of warm global ischemia followed by 20 minutes of reperfusion in the absence (Control group) and presence (NO group) of $4 \mu \mathrm{M}$ NO. In further experiments, the endothelial glycocalyx was enzymatically degraded by means of heparinase followed by reperfusion without (HEP group) and with NO (HEP+NO group).
\end{abstract}

Results Ischemia and reperfusion severely damaged the endothelial glycocalyx. Shedding of heparan sulfate and damage assessed by electron microscopy were less in the presence of
NO. Compared with baseline, coronary fluid extravasation increased after ischemia in the Control, $H E P$, and $H E P+N O$ groups but remained almost unchanged in the $N O$ group. Tissue edema was significantly attenuated in this group. Coronary vascular resistance rose by $25 \%$ to $30 \%$ during reperfusion, but not when NO was applied, irrespective of the state of the glycocalyx. Acute postischemic myocardial release of lactate was comparable in the four groups, whereas release of adenine nucleotide catabolites was reduced $42 \%$ by NO. The coronary venous level of uric acid, a potent antioxidant and scavenger of peroxynitrite, paradoxically decreased during postischemic infusion of NO.

Conclusion The cardioprotective effect of NO in postischemic reperfusion includes prevention of coronary vascular leak and interstitial edema and a tendency to forestall both no-reflow and degradation of the endothelial glycocalyx.

\section{Introduction}

Myocardial damage and coronary microvascular dysfunction, including the no-reflow phenomenon and edema formation, evolve from coronary occlusion and consecutive reperfusion. These are relatively common clinical occurrences, for example, in conjunction with percutaneous coronary angioplasty (PTCA), coronary artery bypass grafting (CABG), and heart transplant reperfusion [1]. Over the past 20 years, major advances have been made toward understanding the role of nitric oxide (NO) in the ischemic biology of the heart and it has become clear that $\mathrm{NO}$, either endogenous or exogenous, rep- resents one of the most important defenses against myocardial ischemia and reperfusion injury. The influence of NO on microvascular permeability is less clear because conflicting results exist in the literature $[2,3]$.

A healthy vascular endothelium is coated by extracellular domains of a large variety of membrane-bound molecules that, together, constitute the glycocalyx. In vivo, the glycocalyx binds plasma proteins, forming the endothelial surface layer. This zone has a thickness of 400 to $500 \mathrm{~nm}$ in microvessels (in some regions, it is even thicker than the endothelial cells 
themselves) and is an integral part of the circulation. Various experimental models showed this large structure to be fundamentally involved in numerous physiological and pathophysiological actions in the circulation. Loss of coronary glycocalyx integrity is accompanied by leakage of fluid from the vascular compartment, resulting in swelling of the pericapillary interstitial space [4-6]. Moreover, it is recognized that the endothelial glycocalyx is especially prone to injury during ischemia/reperfusion $[4,7,8]$. Very recently, damage of the endothelial glycocalyx could be demonstrated in patients undergoing global or regional ischemia during vascular surgery [9]. Perturbation of the glycocalyx also results in adhesion of platelets and leukocytes to the capillary and venular endothelial surface $[10,11]$. Furthermore, coronary dilatation induced by shear stress also depends on an intact endothelial glycocalyx [12]. Based on these findings, there is little doubt that degradation of the endothelial glycocalyx leads to impaired regulation of organ blood flow, to activation of coagulatory and inflammatory pathways, and to tissue edema. Investigating strategies for protecting the endothelial glycocalyx against disruption obviously is an important topic for research.

Postischemic injury appears to depend substantially on the increased production of oxygen-derived free radicals upon reoxygenation. Redox stress may activate proteases, especially metalloproteases. Also, redox stress associated with ischemia/reperfusion induces mast cell degranulation, liberating tryptase and heparanase activity [13]. Activation and liberation of such enzymes will undoubtedly mediate disruption of the endothelial glycocalyx. Thus, by acting as a radical scavenger, NO administered in small amounts during reperfusion might help to protect the endothelium.

Given the established facts above, the present study investigated the effect of exogenous NO, given at the time of reperfusion, on the functional and metabolic outcome following myocardial ischemia/reperfusion, particularly with regard to the state of the endothelial glycocalyx. All experiments were conducted on an intact vascular bed, namely the coronary system of isolated perfused hearts (guinea pig Langendorff preparations), with a highly standardized protocol of ischemia and reperfusion. The impact of exogenous administration of NO (4 $\mu \mathrm{M})$ was investigated by measuring coronary vascular resistance and net fluid filtration with and without enzymatic degradation of the glycocalyx by means of application of heparinase. The rationale for using heparinase is that it has proven to be specific for shedding heparan sulfates and that these structures comprise the greatest portion of the luminal endothelial glycocalyx [14]. The state of the glycocalyx was assessed by electron and light microscopy. The metabolic influence of NO was examined by measuring myocardial release of lactate, catabolism of adenine nucleotides, and degradation of the endogenous antioxidant uric acid during reperfusion.

\section{Materials and methods}

This investigation conforms with the Guide for the Care and Use of Laboratory Animals [15]. Licensure and approval of the investigation were obtained from the Government of Upper Bavaria (file no. 209.1/211-2531.3-3/99).

\section{Heart preparation}

In brief, guinea pigs (male; weight 250 to $300 \mathrm{~g}$ ) were stunned by neck dislocation with a specially designed instrument, and, immediately after the opening of the thorax, the hearts were arrested with ice-cold isotonic saline. Quickly, the aorta was cannulated and the coronaries were perfused in situ at a constant flow rate of $6.0 \mathrm{~mL} /$ minute with a modified Krebs-Henseleit buffer (116 mM NaCl, $23 \mathrm{mM} \mathrm{NaHCO}_{3}, 3.6 \mathrm{mM} \mathrm{KCl}, 1.16$ $\mathrm{mM} \mathrm{KH}_{2} \mathrm{PO}_{4}, 1.2 \mathrm{mM} \mathrm{CaCl}_{2}, 0.58 \mathrm{mM} \mathrm{MgSO}_{4}, 5.4 \mathrm{mM}$ glucose, $0.3 \mathrm{mM}$ pyruvate, and $2.8 \mathrm{U} / \mathrm{L}$ insulin) oxygenated with $94.5 \% \mathrm{O}_{2}$ and $5.5 \% \mathrm{CO}_{2}$ at $37^{\circ} \mathrm{C}, \mathrm{pH} 7.40 \pm 0.05$. Hearts were removed from the thorax and prepared especially for quantifying net coronary fluid leak as described previously $[5,6,16,17]$. The hearts were not inverted and beat spontaneously. Such hearts are not readily suited for determining contractile function. Coronary perfusion pressure in the aortic feed line was registered online (pressure transducer; SeeheimOber Beerbach, Engelsbach, Germany). Coronary venous effluent was collected from the pulmonary artery, cannulated and ending with a slightly negative hydrostatic pressure to preclude coronary venous congestion. Interstitial and lymphatic fluids formed by net filtration appeared at the epicardial surface and dripped off the apex of the heart. This so-called transudate was collected at 1-minute intervals and quantified by weighing on a precision scale. This value was subsequently divided by the ventricular wet weight (w.w.) so that the unit of transudate formation is expressed as milliliter per minute per gram of w.w. Samples of effluent and transudate were then immediately frozen.

Infusion lines were inserted into the aortic feed line just above the coronary ostia to infuse small aliquots of a saturated aqueous solution of NO (see below) or of heparinase type-1 enzyme (Sigma-Aldrich, St. Louis, MO, USA). We have recently shown by electron microscopy, immunohistological staining, and quantitative measurement of constituent parts of the glycocalyx that this enzyme selectively sheds heparan sulfate from the endothelial glycocalyx [14]. Enzymograms (PAGE, gelatine) showed that heparinase had no collagenase activity versus control, excluding enzyme activity, for example, against the basement membrane. Constant-flow instead of constant-pressure perfusion was chosen for the current experimental model to guarantee constant levels of infused substances. The protocol allowed infusion of NO stock solution calculated to yield an arterial infusate concentration of $4 \mu \mathrm{M}$.

\section{Control experiments}

The heart preparations were first characterized without ischemia. Time control experiments were performed to evalu- 
Table 1

\section{Control experiments without ischemia}

\begin{tabular}{lccc} 
& Time $_{\text {Control }}(\mathrm{n}=5)$ & $N_{\text {Control }}(\mathrm{n}=5)$ & $H E P_{\text {Control }}(\mathrm{n}=6)$ \\
\hline Coronary perfusion pressure, percentage & $114 \pm 7$ & $89 \pm 4$ & $119 \pm 5$ \\
Transudate formation, percentage & $128 \pm 17$ & $99 \pm 14$ & $260 \pm 14^{\mathrm{a}}$ \\
\hline
\end{tabular}

Values are presented as a percentage of basal and as mean \pm standard deviation. The groups are as defined in Materials and methods. aP $<0.05$, intragroup difference versus basal.

ate the influence of 15-minute equilibration and 20-minute perfusion without ischemia on coronary perfusion pressure and on transudate formation (Time Control $_{\text {group). Moreover, time- }}$ matched control values were measured after 20-minute administration of $4 \mu \mathrm{M} \mathrm{NO}$ ( $\mathrm{NO}_{\text {Control }}$ group) and after 15minute application of $10 \mathrm{U}$ of heparinase ( $\mathrm{Hep}_{\text {Control }}$ group). The results are presented in Table 1. NO infused in this manner did not elicit vasodilatation; the threshold value required to do so was approximately $10 \mu \mathrm{M}$ (nominal arterial infusate concentration). The application of heparinase caused a measurable increase in transudate formation.

\section{Experimental protocols}

Hearts were randomly assigned among the experimental groups. Figure 1 illustrates the protocols applied to four groups of hearts. The standard condition was that of constant coronary flow perfusion $(6.0 \mathrm{~mL} /$ minute $)$ at $37^{\circ} \mathrm{C}$. After an equilibration period of 15 minutes, hearts were subjected to 15 minutes of global, normothermic, stopped-flow ischemia and then reperfused under the same condition as before ischemia. To maintain the temperature of the preparation during ischemia, hearts were immersed in warm Tyrode's buffer $\left(37^{\circ} \mathrm{C}\right)$. Hearts were reperfused in the absence of NO (Figure 1 , Control group), in the presence of $4 \mu \mathrm{M} N O$ (NO group), and after the glycocalyx had been enzymatically degraded by means of heparinase (10 U of enzyme in a volume of $1.5 \mathrm{~mL}$ applied into the coronaries in the course of the 15-minute ischemic phase), both without NO (HEP group) or with $4 \mu \mathrm{M}$ $\mathrm{NO}(H E P+N O$ group). Samples of transudate and coronary effluent were taken at baseline and during reperfusion. At the end of each experiment, hearts were removed from the perfusion system and the atria and large vessels cut away. Excess surface and intraventricular fluid was swabbed off and the ventricles weighed at once.

Figure 1

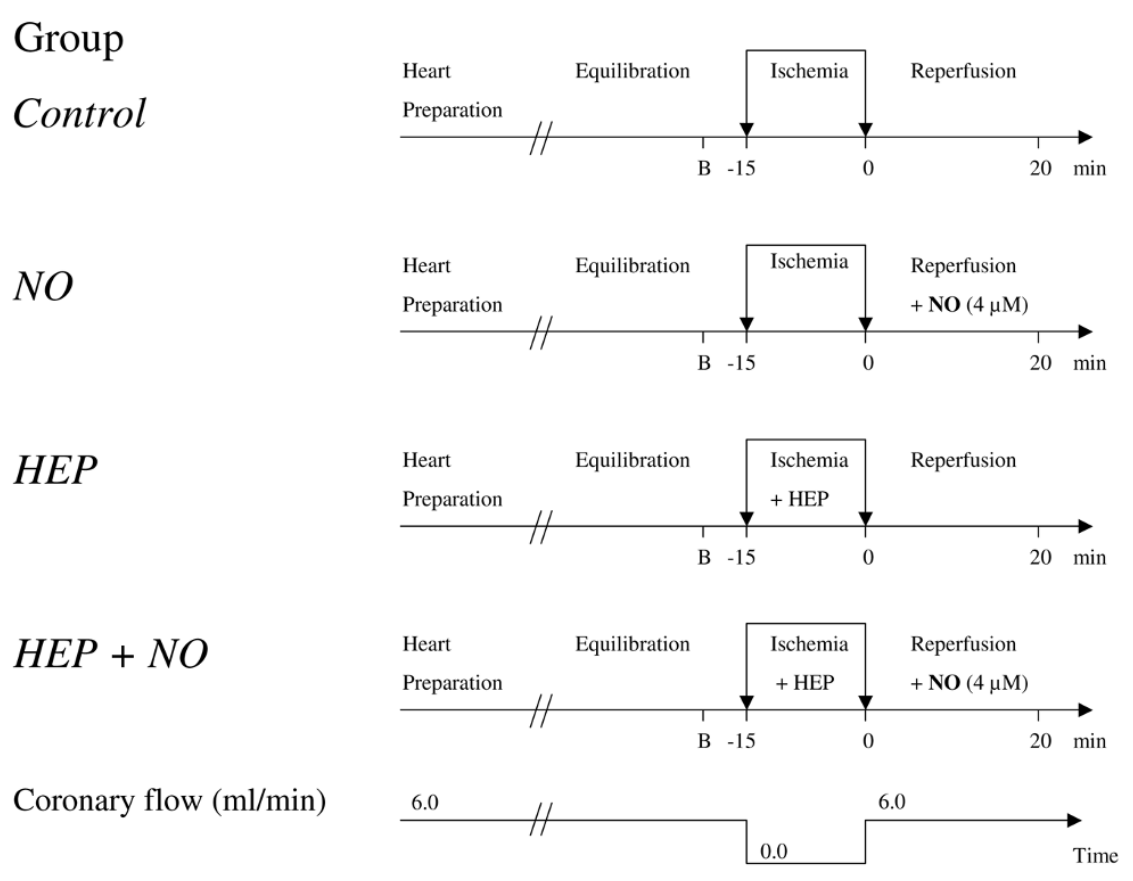

Experimental protocols. After heart preparation and an equilibration period of 15 minutes, hearts were subjected to 15 minutes of global normothermic ischemia followed by 20 minutes of reperfusion. Hearts were reperfused in the absence (Control group) and presence (NO group) of $4 \mu \mathrm{M}$ nitric oxide. In two further series, the glycocalyx was enzymatically degraded by heparinase, applied into the coronary system in the course of ischemia, followed again by reperfusion in the absence (HEP group) and presence (HEP+NO group) of $4 \mu \mathrm{M} N O$. Transudate and coronary effluent were quantified at baseline (B) (just before ischemia) and at $1,2,3,4,5,8,15$, and 20 minutes of reperfusion. HEP, heparinase. 


\section{Preparing and applying NO stock solution}

To prepare an aqueous saturated solution of $\mathrm{NO}, 3 \mathrm{~mL}$ of deionized water was sealed into a small glass vial with a stopper and a long syringe needle was inserted, extending almost to the base of the vial. An additional shorter needle that did not immerse into the solution was inserted and acted as a pressure relief. After the system was purged with argon (Linde AG, Munich, Germany) for a period of 30 minutes at a moderate flow rate, the system was bubbled for 30 minutes with pure NO (Linde AG). Subsequently, the needles were removed and the rubber stopper sealed spontaneously. Just before the experiment, this solution was transferred under exclusion of oxygen into a glass syringe equipped with a long metal cannula. The concentration of $\mathrm{NO}$ resulting from saturation at $20^{\circ} \mathrm{C}$ is approximately $1.91 \mathrm{mM}$.

\section{Quantification of tissue edema}

At the end of each protocol, tissue edema was assessed by measuring ventricular wet weight (at once) and dry weight (after 24 hours of drying at $60^{\circ} \mathrm{C}$ ). The quotient 'wet weight/ dry weight' was then calculated.

\section{Determination of release of lactate, purines, and uric acid}

Coronary venous effluent of the Control and NO groups was collected via the pulmonary artery, and aliquots were stored ($20^{\circ} \mathrm{C}$ ) until analyzed. The concentration of lactate was determined by high-performance liquid chromatography (HPLC) in aliquots acidified $(\mathrm{pH} 2.0)$ with perchloric acid [18]. The total concentrations of the purines adenosine, inosine, hypoxanthine, xanthine, guanosine, and urate together (products of adenine nucleotide catabolism) as well as that of uric acid alone were quantified by reverse-phase HPLC [19]. In each case, the concentration value, multiplied by the coronary flow rate and divided by the ventricular w.w., yielded the respective rate of cardiac release per gram of heart weight. The cumulative release of purines was established over the first 15 minutes of reperfusion by calculating an area under the curve using the trapezoidal method (Sigma plot; SPSS Inc., Chicago, IL, USA).

\section{Determination of heparan sulfate}

After 15 minutes of ischemia, samples of effluent were collected at $5,8,15$, and 20 minutes during reperfusion and were used for assessing shedding of heparan sulfate. To aggravate the ischemia/reperfusion injury, additional hearts were subjected to 20 minutes of ischemia followed by 40 minutes of reperfusion. All samples were preconcentrated over $10-\mathrm{kDa}$ cutoff membrane filters (Millipore, Eschborn, Germany). Concentrations were determined with an enzyme-linked immunosorbent assay (Seikagaku Corporation, Tokyo, Japan). The cumulative release was established by calculating an area under the curve (see above).

\section{Light microscopy and immunohistochemistry}

Hearts were perfusion-fixed after minimal perfusion $\quad<1$ minute), after 15 minutes of ischemia followed by 20 minutes of reperfusion in the absence and presence of $\mathrm{NO}$, and after the glycocalyx had been enzymatically degraded by means of heparinase in the course of the ischemic phase. Fixation occurred by the addition of formaldehyde to the flowing KrebsHenseleit buffer to a resulting concentration of $1 \%$. After 4 minutes, the hearts were removed from the apparatus and stored in 4\% formaldehyde solution for 24 hours. Paraffin sections $(5 \mu \mathrm{m})$ were immunohistochemically stained with monoclonal antibody against heparan sulfate (Seikagaku Corporation). The primary antibody, applied to generate an avidin-biotin horseradish peroxidase complex with the Vectastain kit (Vector Laboratories, Burlingame, CA, USA), was diluted and handled as follows: anti-heparan sulfate 1:100, tissue preincubation with $0.2 \%$ trypsin at $37^{\circ} \mathrm{C}$. Controls in which the primary antibody was replaced with buffer were treated identically. Diaminobenzidine or aminoethylcarbazole was used as chromogen.

\section{Electron microscopy}

Electron microscopy, performed in modification of Vogel and colleagues [20], was based on in situ stabilization of the glycocalyx by intracoronary application of a fixative containing lanthanum ions and glutaraldehyde $[5,6,16,17]$.

\section{Statistical analysis}

Because the measured data were distributed normally (determined by Kolmogorov-Smirnov test), these are presented as mean \pm standard deviation unless otherwise indicated. Comparisons were made using analysis of variance for repeated measurements. Post hoc testing was performed using the Student-Newman-Keuls method for multiple comparisons. A $P$ value of less than 0.05 was considered to be significant.

\section{Results}

The effect of ischemia and reperfusion on coronary perfusion pressure is shown in Figure 2 for all experimental groups. In the Control and HEP groups, the data reveal that a transient postischemic vasodilatation (decrease in coronary perfusion pressure) tended to develop approximately 5 minutes after the end of ischemia. This was followed by vasoconstriction (increase in coronary perfusion pressure). After 20 minutes of reperfusion, coronary perfusion pressure had increased significantly versus baseline in the Control group. In the presence of $4 \mu \mathrm{M} \mathrm{NO}$ (NO and HEP+NO groups), this latter increase in coronary perfusion pressure did not occur. Although there were no significant intergroup differences after 20 minutes, there was a significantly lower coronary resistance for all hearts with $\mathrm{NO}$ as compared with those without $(P<0.05$, pooled data, $n=10$ and 11 , respectively). Pertinently, coronary resistance did not increase within the first 5 minutes when the infusion of $\mathrm{NO}$ was terminated after 20 minutes (results 
not shown). Thus, there was no acute and direct dilatatory effect of NO.

Postischemic changes in transudate formation (that is, of net fluid filtration in the intact coronary bed of isolated guinea pig hearts) are illustrated in Figure 3 for all experimental groups. Baseline transudate formation - about 20 to $50 \mu \mathrm{L} /$ minute per gram of w.w. - did not differ significantly among the four groups. Postischemically, there was an increase in transudate formation in the Control, $H E P$, and $H E P+N O$ groups with respect to the individual group baseline $(\Delta=+50,+85$, and $+44 \mu \mathrm{L} /$ minute per gram of w.w. after 20 minutes, respectively). In contrast, when shedding of heparan sulfate had not been enzymatically induced prior to reperfusion (see below), transudate formation remained almost unchanged when $4 \mu \mathrm{M}$ $\mathrm{NO}$ was applied during reperfusion $(\Delta=-13 \mu \mathrm{L} /$ minute per gram of w.w. after 20 minutes) and was significantly lower than in the Control group at all times of reperfusion (Figure 3). Interestingly, no correlation was found between transudate formation and coronary perfusion pressure $\left(R^{2}=0.016 ; n=189\right.$, pooled values of 21 hearts at 9 measuring time points each), also demonstrating that changes in transudate formation were not the result of any putative acute vasodilatatory effect of NO.

Mean wet-to-dry weight ratios of the isolated heart preparations determined at the end of each experimental protocol are listed in Table 2. The mean ratios for hearts of Control, HEP, and $H E P+N O$ groups ranged from 7.9 to 8.3. NO applied to

Figure 2

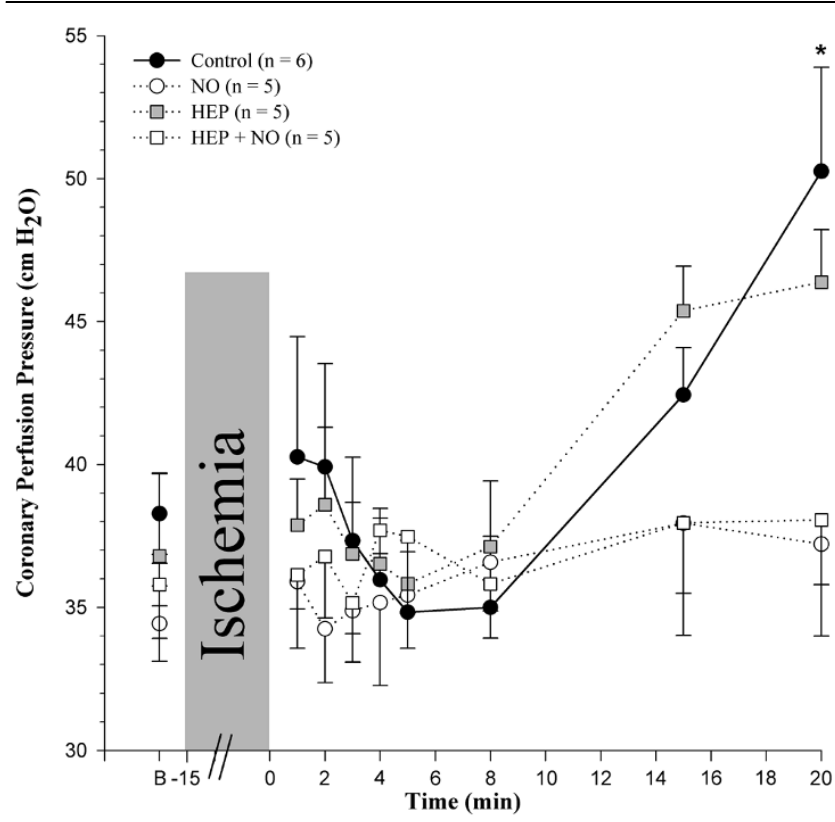

Effect of ischemia/reperfusion on coronary perfusion pressure. Groups are as defined in the legend of Figure 1. Values are presented as mean \pm standard error of the mean. ${ }^{\star} P<0.05$, intragroup difference versus basal (B).
Figure 3

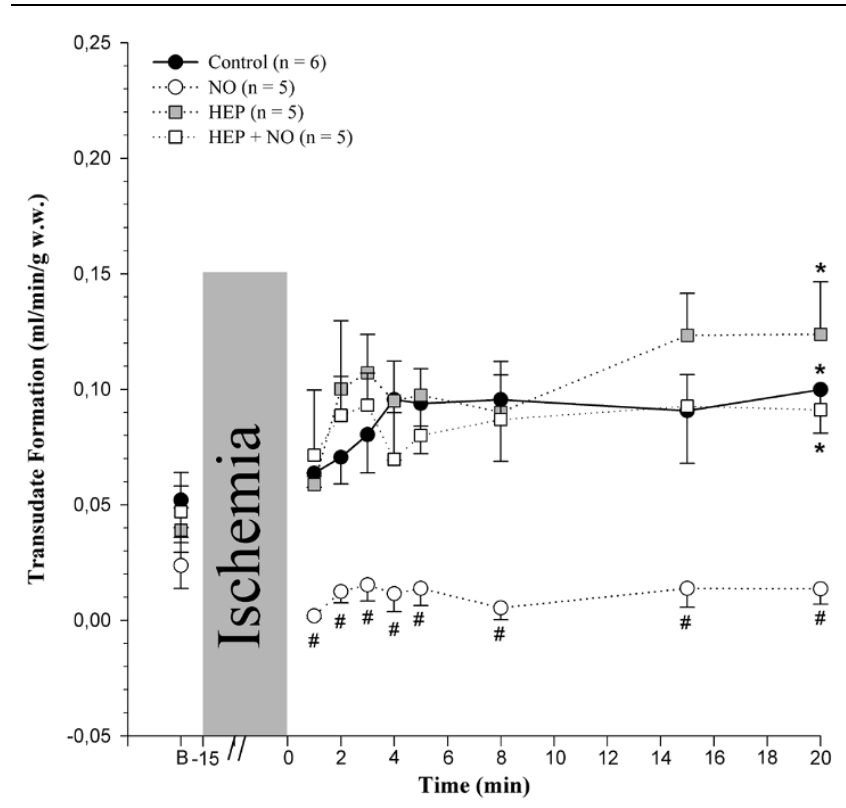

Effect of ischemia/reperfusion on coronary transudate formation. The groups are as defined in the legend of Figure 1. Values are presented as mean \pm standard error of the mean. ${ }^{*} P<0.05$, intragroup difference versus basal $(\mathrm{B}) ; \# P<0.05$, intergroup difference versus the Control group. w.w., wet weight.

group) yielded a mean ratio of 6.9 , indicating a significant decrease in tissue edema formation.

Metabolic data were determined in the absence (Control group) and presence (NO group) of $4 \mu \mathrm{M} N O$. The release of purine compounds in the coronary effluent serves as a quantitative measure of adenine nucleotide catabolism in the heart and is illustrated in Figure 4a. A beneficial effect of NO was observed: acute postischemic release of purines was reduced in its presence and cumulative purine release during reperfusion was almost halved (Control group: area under the curve $=1,907 \pm 517 \mathrm{nmol}$ per gram of w.w.; NO group: area under the curve $=1,097 \pm 427 \mathrm{nmol}$ per gram of w.w.; $P<$ $0.05, n=6$ and 5 , respectively). This effect exceeded by far a decrease in the net amount of urate released from these hearts: postischemic release of uric acid (Figure 4b) paralleled that of total purines in the Control group, increasing more than eightfold during early reperfusion. However, it accounted for only about $10 \%$ of the total release of purines in the Control group. In the presence of $\mathrm{NO}$, urate decreased significantly to only a few percent of the total purine release throughout. Lactate release (Figure 4c) increased more than 10-fold during early reperfusion in both the Control and NO groups and was not significantly changed by the presence of $\mathrm{NO}$ during the subsequent reperfusion. There was no difference in the cumulative release of lactate (area under the curve) between both groups. 
Table 2

\begin{tabular}{ll}
\hline Quantification of tissue edema & \\
\hline Group & Wet weight/dry weight \\
\hline Control experiments & \\
Time $_{\text {Control }}(n=5)$ & $7.0 \pm 0.7$ \\
$N O_{\text {Control }}(n=5)$ & $7.0 \pm 0.6$ \\
$H E P_{\text {Control }}(n=6)$ & $8.5 \pm 0.6$ \\
Experimental groups & \\
Control $(n=6)$ & $8.0 \pm 0.3$ \\
$N O(n=5)$ & $6.9 \pm 0.2^{a}$ \\
$H E P(n=5)$ & $8.3 \pm 0.6$ \\
$H E P+N O(n=5)$ & $7.9 \pm 0.6$ \\
\hline
\end{tabular}

Values are presented as mean \pm standard deviation. ${ }^{a} P<0.05$ with respect to Control, HEP, and $H E P+N O$ groups.

Light microscopy after immunohistochemical staining of nonischemic hearts evidenced heparan sulfate as a component of the endothelial glycocalyx (Figures $5 \mathrm{a}$ and $5 \mathrm{~b}$, respectively). In hearts subjected to ischemia and reperfusion without $\mathrm{NO}$, the staining of the endothelial cell lining was markedly reduced (Figures $5 c$ and $5 d$, respectively). In hearts that were reperfused after the glycocalyx had been enzymatically degraded by means of heparinase, no heparan sulfate was detected at the endothelial cell lining (Figures $5 e$ and $5 f$, respectively). Hearts reperfused in the presence of $\mathrm{NO}$ showed some positive staining for heparan sulfate after 20 minutes of reperfusion (Figures $5 \mathrm{~g}$ and $5 \mathrm{~h}$, respectively). Interestingly, the walls of the vasculature also contained heparan sulfate.

Material immunopositive for heparan sulfate was detected in the coronary effluent of the isolated perfused hearts and a postischemic increase was observed after 15 minutes of ischemia (Figure 6a). There was a tendency toward a lower cumulative release of heparan sulfate during reperfusion in the presence of NO but this lacked statistical significance. However, extending the ischemic period to 20 minutes followed by 40 minutes of reperfusion showed a significantly lower cumulative release of heparan sulfate during reperfusion in the presence of NO.

Exemplary electron microscopic images illustrating coronary vessels of the isolated guinea pig hearts are shown in Figure 7. In comparison with a nonischemic heart, where an endothelial glycocalyx of approximately 200- to $300-\mathrm{nm}$ thickness was found in the coronary vasculature, hearts subjected to ischemia and reperfusion showed a dramatic loss of the endothelial surface lining and strong edema formation (Figures $7 a$ and $7 b$, respectively). Hearts that had received NO during reperfusion exhibited less edema, but the glycocalyx still showed partial denudation (Figure 7c). Hearts pretreated with heparinase during ischemia were completely denuded, irrespective of the
Figure 4
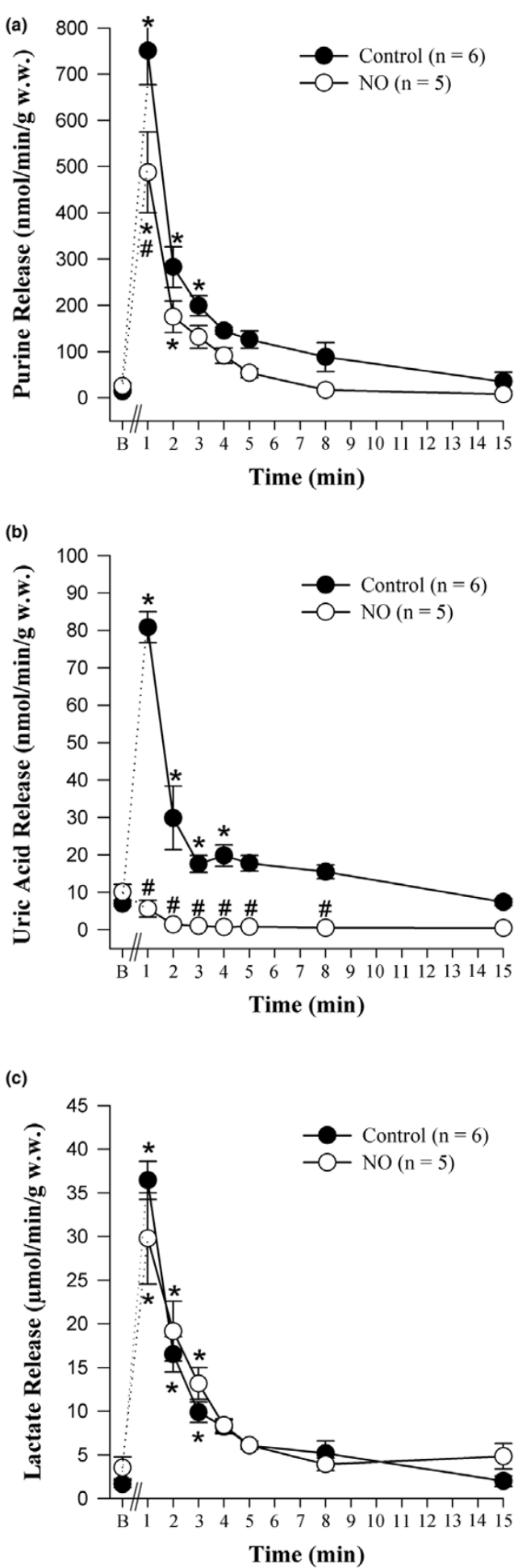

Coronary venous release of metabolites during the first 15 minutes of myocardial reperfusion. (a) Release of purines. (b) Release of urate. (c) Release of lactate. Hearts were reperfused in the absence (Control group) and presence (NO group) of $4 \mu \mathrm{M}$ nitric oxide. Values are presented as mean \pm standard error of the mean. ${ }^{*} P<0.05$, intragroup difference versus basal $(\mathrm{B}) ;{ }^{\#} P<0.05$, intergroup difference versus the Control group. w.w., wet weight. 
Figure 5
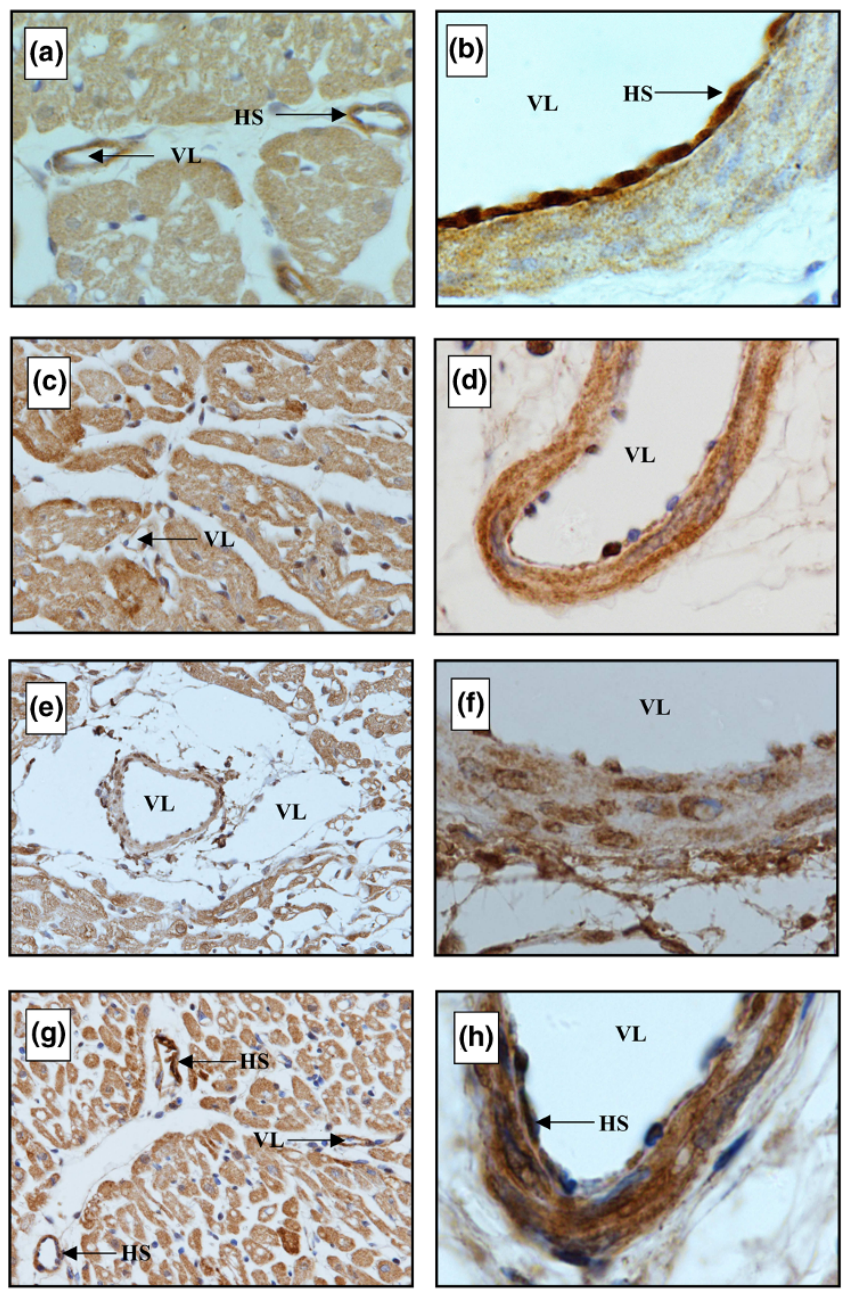

Immunostaining of microvessels of guinea pig hearts with monoclonal antibody against heparan sulfate after minimal perfusion (a, b), after 15 minutes of ischemia followed by 20 minutes of reperfusion in the absence $(\mathbf{c}, \mathbf{d})$ and presence $(\mathbf{g}, \mathbf{h})$ of nitric oxide, and after the glycocalyx had been enzymatically degraded by means of heparinase $(\mathbf{e}, \mathbf{f})$. Specimens are paraffin-embedded sections of guinea pig heart. Original magnifications: $\times 20$ (lefthand panels) and $\times 100$ (righthand panels). HS, heparan sulfate; VL, vascular lumen.

presence of $\mathrm{NO}$ during reperfusion (Figures $7 \mathrm{~d}$ and $7 \mathrm{e}$, respectively).

\section{Discussion}

The major findings of this study are that $\mathrm{NO}$, applied with the onset of reoxygenation, protects isolated hearts against some facets of postischemic reperfusion injury and that this protective effect seems to extend to the endothelial glycocalyx in the coronary vascular bed. The evidence provided by this investigation is the following:

1. Coronary resistance tended to increase during reperfusion, but not if NO was applied. This effect seemed to be maintained even if the glycocalyx had been degraded beforehand.
Figure 6
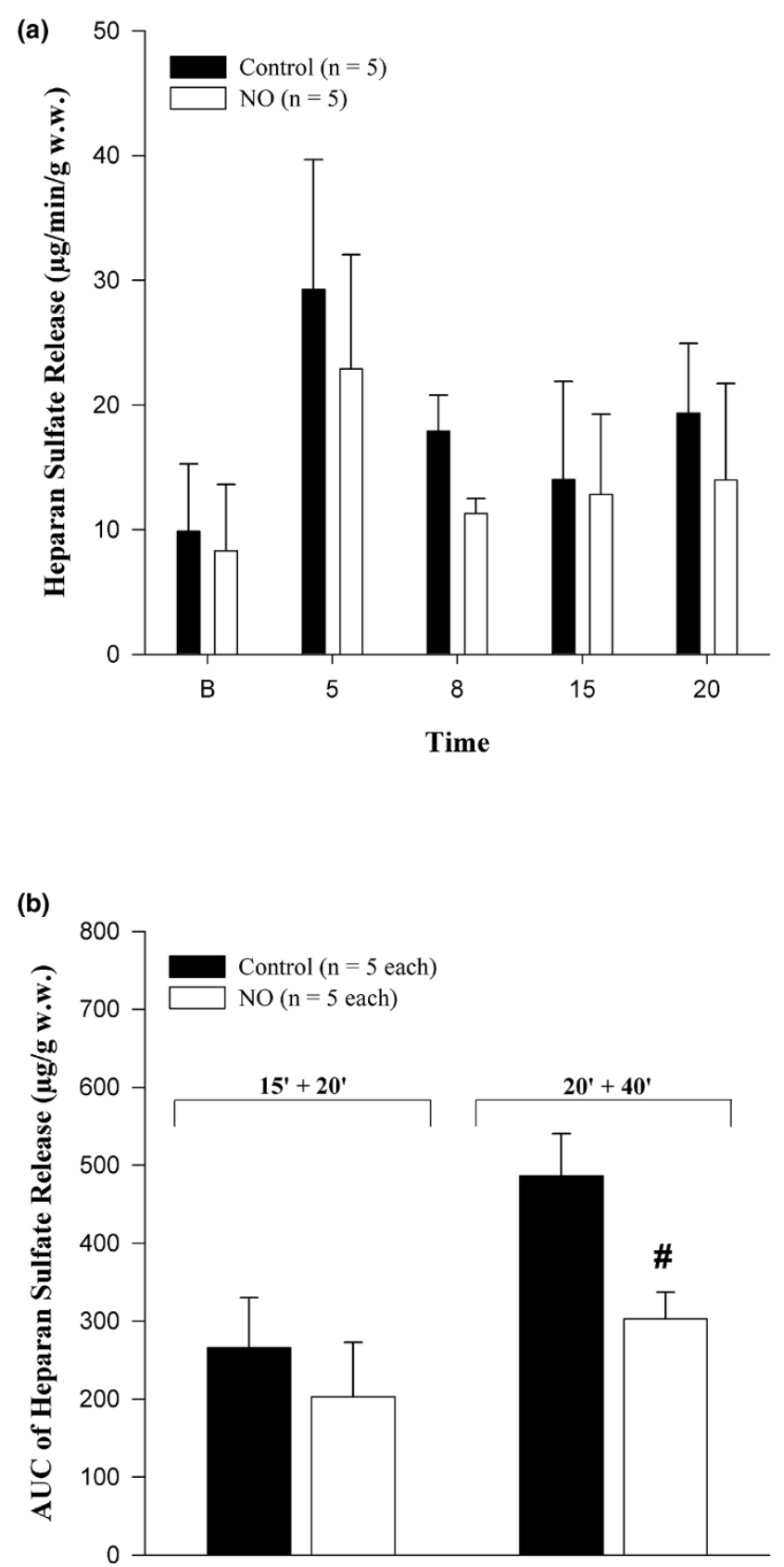

Heparan sulfate release in coronary venous effluent. (a) Absolute release of heparan sulfate in hearts subjected to 15 minutes of ischemia followed by 20 minutes of reperfusion. (b) Cumulative release of heparan sulfate in hearts subjected to 15 minutes of ischemia followed by 20 minutes of reperfusion $\left(15^{\prime}+20^{\prime}\right)$ and in hearts subjected to 20 minutes of ischemia followed by 40 minutes of reperfusion $\left(20^{\prime}+\right.$ 40 '). Hearts were reperfused in the absence (Control group) and presence (NO group) of $4 \mu \mathrm{M}$ nitric oxide. Values are presented as mean \pm standard error of the mean. ${ }^{\#} P<0.05$, intergroup difference versus the Control group. AUC, area under the curve; B, baseline; w.w., wet weight. 


\section{Figure 7}
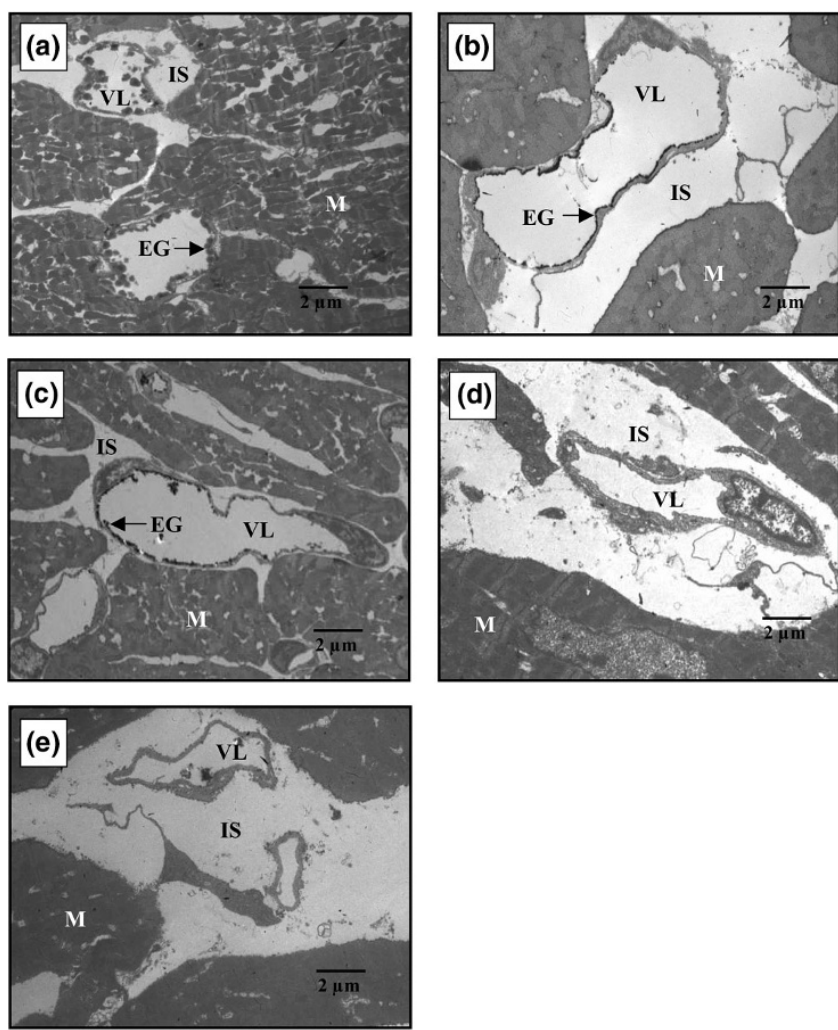

Exemplary electron microscopic images of hearts without ischemia (a) and hearts subjected to 15 minutes of warm ischemia and 20 minutes of reperfusion without nitric oxide (NO) (b) (Control group) and with 4 $\mu \mathrm{M}$ exogenous NO (c) (NO group). The glycocalyx of 200 to $300 \mathrm{~nm}$ in width normally present in all vessels has virtually disappeared after ischemia and reperfusion in the absence of $\mathrm{NO}$ and edema has developed. NO partly mitigated these effects. The effect of heparinase is shown in (d) (HEP group) and (e) (HEP+NO group). EG, endothelial glycocalyx; IS, interstitial space; M, cardiomyocytes; VL, vascular lumen.

2. Fluid extravasation in the coronary system was increased after ischemia, but not if NO was applied during reperfusion. The postischemic increase in tissue edema was alleviated if hearts were reperfused in the presence of NO. NO had no preventive effect on coronary leak or edema if the endothelial glycocalyx was degraded beforehand.

3. NO attenuated shedding of the endothelial glycocalyx, as judged by enzyme-linked immunosorbent assay and electron microscopy.

4. Metabolic stress was mitigated during reperfusion with NO, as evidenced by a significantly lowered catabolism of highenergy adenine nucleotides.

The observed trends in change of coronary perfusion pressure comply with the no-reflow phenomenon and may be explained in part by the changing absolute availability of endogenous $\mathrm{NO}$ during reperfusion. The concentration of endogenous $\mathrm{NO}$ in the coronary effluent, about $0.2 \mu \mathrm{M}$ during normoperfusion [21,22], increases again from zero during early reperfusion [23]. However, with prolongation of reperfusion, NO synthase activity decreases and $\mathrm{NO}$ is also consumed by reaction with superoxide and hydroxyl radicals. Thus, NO decreases below baseline values [22]. These expected changes in NO concentration parallel the changes in coronary perfusion pressure observed in the present study: hearts reperfused in the absence of NO (Control and HEP groups) showed an intermittent decrease in coronary perfusion pressure followed by an increase. Such an increase did not occur in hearts reperfused with exogenous $N O$ (NO and $H E P+N O$ groups), although $\mathrm{NO}$ applied into the arterial perfusate at this level (4 $\mu \mathrm{M})$ did not per se elicit coronary dilatation. Since coronary perfusion pressure directly reflects coronary resistance under conditions of constant coronary flow as used here, it seems that moderate amounts of exogenous NO (see below) may be able to compensate the increase in vascular tone caused by depletion of endogenous NO in reperfused hearts. However, alternative actions, not directed immediately toward the vascular smooth muscle, also need to be considered. One such possibility would be alleviation of interstitial edema (that is, of extramural compression). This was found only for the NO and not for the $H E P+N O$ hearts, suggesting that interstitial pressure was not a major determinant of coronary resistance. Despite the seemingly good concordance of effect and expectation concerning NO levels, the actual magnitudes of the coronary resistance changes seen in the present study were definitely limited. Thus, the action of NO in this respect should not be overstated.

A far stronger action was evident concerning vascular leak. In the isolated perfused heart model, changes in transmural fluid passage in the entire coronary bed can be assessed directly by determining the flow of transudate. This fluid, which appears on the epicardial surface, may be regarded as a mixture of interstitial and lymphatic liquid and its flow is equal to net fluid filtration in the coronary system. The basal value of up to $50 \mu \mathrm{L} /$ minute per gram of w.w. obtained in the present study compared favorably with that reported by others $[24,25]$, as did the observed postischemic increase. The endothelial glycocalyx plays a major role in the development of postischemic coronary vascular leak $[8,26]$ and, together with the endothelial cells themselves, constitutes the vascular 'double barrier' against fluid extravasation $[16,17]$.

Bulk permeability of water and small hydrophilic solutes is basically influenced by the Starling forces of intravasal and extravasal hydrostatic and oncotic pressures and by the vascular barrier. The only variable force in our model was the coronary perfusion pressure. However, there was certainly no consistent relationship between coronary perfusion pressure and transudate formation in the four groups of hearts (compare Figures 2 and 3 ). Thus, the observed differences of net fluid transport across the vascular wall at any one time 
must reflect differences in hydraulic conductivity (that is, in the integrity of the double barrier composed of the endothelial glycocalyx and the endothelial cells). When the glycocalyx is damaged, vascular permeability increases. This was seen in hearts treated with heparinase (Table 1: HEP Control; Figure 2: $H E P$ and $H E P+N O$ groups). The elevated formation of transudate after 15 minutes of ischemia in hearts not treated with heparinase (Control group) supports the view that ischemia/ reperfusion damaged the glycocalyx. In agreement with the assumption that $\mathrm{NO}$ alleviated degradation of the coronary endothelial glycocalyx, there was less leak and less heparan sulfate-reactive material tended to appear in the coronary effluent of those hearts reperfused solely with $4 \mu \mathrm{M}$ NO. Electron microscopic visualization of the microvasculature in control hearts and hearts subjected to ischemia/reperfusion without and with NO corroborated the partial protection of the endothelial glycocalyx by exogenous NO (Figure 7).

There has been an ongoing debate in the literature as to whether NO is a protectant or a source of injury in the reperfused heart, but it has clearly been demonstrated that NO has infarct-sparing abilities in isolated buffer-perfused hearts [27]. In line with the results of the present study, various drugs (for example, statins, angiotensin-converting enzyme inhibitors, angiotensin-receptor blockers, nitrates, and nitrites) have produced beneficial effects in experimental models of ischemia/ reperfusion via the enhancement of $\mathrm{NO}$ bioavailability [28]. Cardioprotection by inhalational NO has not been conclusively demonstrated in humans. However, the NO donor nitroprusside improved coronary flow and myocardial pump function in patients undergoing elective PTCA [29] and CABG [30,31], respectively.

The metabolic data also indicate further protective actions of $\mathrm{NO}$ in the isolated guinea pig hearts. The release of purines has been shown to be a sensitive and accurate index of energy metabolism [32]: purine release increases whenever energy production is impaired or inadequate to meet demand and decreases if energy balance is restored. It may be postulated that NO mitigates energy demand resulting from oxidative stress (for example, the familiar postischemic dysregulation of cytosolic ion levels). Irrespectively, the observed preservation of purines in hearts reperfused with $\mathrm{NO}$ will serve to restore and/or maintain levels of adenine nucleotides and high-energy phosphates because de novo synthesis of purines - the only alternative to salvage - is an energetically very costly process.

In contrast, washout of lactate did not differ between the Control and NO groups. Thus, direct ischemic stress was comparable in the two groups and, as was to be expected, is uninfluenced by NO applied afterwards. This result is in full accord with the study postulate.

Microvascular permeability induced by ischemia/reperfusion in the dog heart was reduced by antioxidants and radical scaven- gers [33]. A protective effect of $\mathrm{NO}$ could reside in an enhanced scavenging of reactive oxygen species, particularly hydroxyl and superoxide radicals. Indirect evidence for such an effect of NO can be derived from the substantially lower concentration of uric acid found in the effluent of hearts reperfused with NO. Uric acid reacts avidly with peroxynitrite formed by reaction of NO with superoxide [18]. The enhanced formation of urate due to nucleotide catabolism in hearts subjected to ischemia/reperfusion (see Control group) actually provides more endogenous antioxidant to mitigate the removal of peroxynitrite. It should be kept in mind that humans have exceptionally high blood urate levels [34]. The generation of physiological antioxidants such as urate in complete biological systems allows NO to alleviate postischemic redox stress without giving rise to toxic products [19,34]. Although the data certainly show a change in the stress to hearts of the NO group as opposed to the Control group (compatible with a scavenging of radicals and reactive oxidative species), the metabolic effects seen in these hearts could also arise from Gprotein- and c-GMP-induced actions or from other cellular effects of NO $[27,28]$.

A critique of the experimental model used here should address the likely partial oxidation of $\mathrm{NO}$ applied into an oxygenated Krebs-Henseleit perfusate. Although the time of contact from the site of infusion to entry into the coronary system is only about 0.2 seconds, some of the $\mathrm{NO}$ will react with oxygen to form toxic nitrogen dioxide $\left(\mathrm{NO}_{2}\right)$ and higher oxides. A similar fate befalls $\mathrm{NO}$ applied via the respiratory tract in human therapy. Like peroxynitrite, $\mathrm{NO}_{2}$ reacts with urate. Thus, part of the marked decrease in coronary urate levels seen in our perfusion protocol upon application of $\mathrm{NO}$ may well reflect oxidative degradation due to inactivation of $\mathrm{NO}_{2}$. Owing to the high plasma levels of urate in humans, the same detoxification may be expected when NO is used clinically, explaining its relative safety in human use [35].

We had deliberated extensively on alternative routes and sources of $\mathrm{NO}$ before deciding on the application of physically dissolved, pure NO. A major drawback of chemical generators of NO, such as NO-penicillamine, S-nitrosoglutathione, and $\mathrm{SIN}-1$, is that only the rate of generation of NO can be predicted, but not the actual concentration in the solution being acutely applied, as hydrolysis to $\mathrm{NO}$ and oxidation of $\mathrm{NO}$ are occurring from the moment water is added to compose the respective stock solutions. On the other hand, the concentration of NO in a saturated aqueous solution at room temperature and normal atmospheric pressure is well characterized in the literature. Moreover, the choice of direct NO generators in clinical use is neither very great nor tempting.

Action on the combined barrier formed by the glycocalyx and the endothelial cell bodies is better assessed in the presence of colloidal substances [6]. Colloids are able to establish an oncotic gradient at the endothelial surface, opposing fluid fil- 
tration [17]. However, postischemic application of hydroxyethyl starch alone to the perfusate already prevented much of the increase in coronary leak that developed during reperfusion [36].

Because the isolated hearts are perfused with blood-free Krebs-Henseleit buffer solution, the endothelial cells are presumably covered only by membrane-anchored molecules. That is, the glycocalyx in its strictest sense, and not the endothelial surface layer, is under investigation. It is also impossible to assess aspects of ischemia/reperfusion injuries that may be initiated and influenced by plasma-borne factors and formed constituents of blood. On the other hand, physiologic antioxidants of plasma are absent, perhaps accentuating damage by reactive oxygen species. Inhalational NO has been shown to be beneficial after myocardial infarction [37], but no link was drawn to the glycocalyx. This is quite understandable because it is not easy to directly visualize the glycocalyx in vivo. Thus, in vitro models are still required.

\section{Conclusion}

In summary, the present study shows a protective role for NO during myocardial reperfusion. The cardioprotective effect of $\mathrm{NO}$ in ischemia/reperfusion includes attenuation of the coronary no-reflow phenomenon and prevention of coronary vascular leak and tissue edema. The latter actions are based on preservation of the endothelial glycocalyx.

\section{Key messages}

- Nitric oxide attenuates the increase in coronary leak and edema formation induced by ischemia/reperfusion.

- This action is based on protection of the endothelial glycocalyx against shedding.

\section{Competing interests}

The authors declare that they have no competing interests.

\section{Authors' contributions}

DB was responsible for acquisition of data, analysis and interpretation of data, and drafting the manuscript. MR conceived of the study and participated in data interpretation and manuscript development. DB and MR contributed equally. MJ and DC took part in all animal experiments performed and were responsible for surgical preparation and data collection. MS and UW performed the electron microscopy, light microscopy, and immunohistochemistry. PC analysed and interpreted the data. BFB was responsible for the study design and the analysis and interpretation of data and helped to draft the manuscript. All authors have read and approved the final manuscript.

\section{Acknowledgements}

The authors gratefully acknowledge the excellent technical assistance of Dora Kiesl and Astrid Sulz. This work was performed using departmental research funding provided by the Government of Bavaria (Bavarian State Ministry of Science, Research, and the Arts, Munich, Germany) and a grant provided by the GEMI Fund (Harvard Medical International, Boston, MA, USA, and Karolinska Institute, Stockholm, Sweden, no. 033/03). This work is attributed to the Clinic of Anesthesiology, LudwigMaximilians-University, Munich, Germany.

\section{References}

1. Reffelmann T, Kloner RA: The no-reflow phenomenon: a basic mechanism of myocardial ischemia and reperfusion. Basic Res Cardiol 2006, 101:359-372.

2. He P, Liu B, Curry FE: Effect of nitric oxide synthase inhibitors on endothelial $\left[\mathrm{Ca}^{2+}\right]_{i}$ and microvessel permeability. $A m$ Physiol 1997, 272:H176-H185.

3. Kubes P, Granger DN: Nitric oxide modulates microvascular permeability. Am J Physiol 1992, 262:H611-H615.

4. Berg BM van den, Vink H, Spaan JA: The endothelial glycocalyx protects against myocardial edema. Circ Res 2003, 92:592-594.

5. Bruegger D, Jacob M, Rehm M, Loetsch M, Welsch U, Conzen P, Becker BF: Atrial natriuretic peptide induces shedding of the endothelial glycocalyx in the coronary vascular bed of guinea pig hearts. Am J Physiol Heart Circ Physiol 2005, 289:H1993-H1999.

6. Jacob M, Bruegger D, Rehm M, Welsch U, Conzen P, Becker BF: Contrasting effects of colloid and crystalloid resuscitation fluids on cardiac vascular permeability. Anesthesiology 2006, 104:1223-1231.

7. Mulivor AW, Lipowsky $\mathrm{HH}$ : Inflammation- and ischemiainduced shedding of venular glycocalyx. Am J Physiol Heart Circ Physio/ 2004, 286:H1672-H1680.

8. Kurzelewski M, Czarnowska E, Beresewicz A: Superoxide-and nitric oxide-derived species mediate endothelial dysfunction, endothelial glycocalyx disruption, and enhanced neutrophil adhesion in the post-ischemic guinea-pig heart. J Physiol Pharmacol 2005, 56:163-178.

9. Rehm M, Bruegger D, Christ F, Conzen P, Thiel M, Jacob M, Chappell D, Stoeckelhuber M, Welsch U, Reichart B, Peter K, Becker BF: Shedding of the endothelial glycocalyx in patients undergoing major vascular surgery with global and regional ischemia. Circulation 2007, 116:1896-1906.

10. Reitsma S, Slaaf DW, Vink H, van Zandvoort MA, oude Egbrink MG: The endothelial glycocalyx: composition, functions, and visualization. Pflugers Arch 2007, 454:345-359.

11. Vink $H$, Constantinescu AA, Spaan JA: Oxidized lipoproteins degrade the endothelial surface layer: implications for platelet-endothelial cell adhesion. Circulation 2000 101:1500-1502.

12. Jacob M, Rehm M, Loetsch M, Paul JO, Bruegger D, Welsch $U$, Conzen $\mathrm{P}$, Becker BF: The endothelial glycocalyx prefers albumin for evoking shear stress-induced, nitric oxide-mediated coronary dilatation. J Vasc Res 2007, 44:435-443.

13. Gilles S, Zahler S, Welsch U, Sommerhoff CP, Becker BF: Release of TNF-alpha during myocardial reperfusion depends on oxidative stress and is prevented by mast cell stabilizers. Cardiovasc Res 2003, 60:608-616.

14. Chappell $D$, Jacob $M$, Rehm $M$, Stoeckelhuber $M$, Welsch $U$, Conzen P, Becker BF: Heparinase selectively sheds heparan sulphate from the endothelial glycocalyx. Biol Chem 2008, 389:79-82.

15. Institute of Laboratory Animal Resources: Guide for the Care and Use of Laboratory Animals Washington, D.C.: National Academy Press; 1996.

16. Rehm M, Zahler S, Lotsch $M$, Welsch $U$, Conzen $P$, Jacob $M$, Becker BF: Endothelial glycocalyx as an additional barrier determining extravasation of $6 \%$ hydroxyethyl starch or $5 \%$ albumin solutions in the coronary vascular bed. Anesthesio/ogy 2004, 100:1211-1223.

17. Jacob $M$, Bruegger $D$, Rehm $M$, Stoeckelhuber $M$, Welsch $U$, Conzen P, Becker BF: The endothelial glycocalyx affords compatibility of Starling's principle and high cardiac interstitial albumin levels. Cardiovasc Res 2007, 73:575-586.

18. Mobert J, Becker BF, Zahler S, Gerlach E: Hemodynamic effects of isoprostanes (8-iso-prostaglandin F2alpha and E2) in iso- 
lated guinea pig hearts. J Cardiovasc Pharmacol 1997, 29:789-794.

19. Becker BF, Reinholz N, Ozcelik T, Leipert B, Gerlach E: Uric acid as radical scavenger and antioxidant in the heart. Pflugers Arch 1989, 415:127-135.

20. Vogel J, Sperandio M, Pries AR, Linderkamp O, Gaethgens P, Kuschinsky W: Influence of the endothelial glycocalyx on cerebral blood flow in mice. J Cereb Blood Flow Metab 2000, 20:1571-1578.

21. Woditsch I, Schror K: Prostacyclin rather than endogenous nitric oxide is a tissue protective factor in myocardial ischemia. Am J Physiol 1992, 263: $\mathrm{H} 1390-\mathrm{H} 1396$.

22. Zahler S, Kupatt C, Becker BF: ACE-inhibition attenuates cardiac cell damage and preserves release of $\mathrm{NO}$ in the postischemic heart. Immunopharmacology 1999, 44:27-33.

23. Wang $P, Z$ weier JL: Measurement of nitric oxide and peroxynitrite generation in the postischemic heart. Evidence for peroxynitrite-mediated reperfusion injury. J Biol Chem 1996, 271:29223-29230.

24. Kupatt C, Zahler S, Seligmann C, Massoudy P, Becker BF, Gerlach E: Nitric oxide mitigates leukocyte adhesion and vascular leak after myocardial ischemia. J Mol Cell Cardiol 1996, 28:643-654.

25. Wienen $W$, Jungling $E$, Kammermeier $\mathrm{H}$ : Enzyme release into the interstitial space of the isolated rat heart induced by changes in contractile performance. Cardiovasc Res 1994, 28:1292-1298.

26. Beresewicz A, Czarnowska E, Maczewski M: Ischemic preconditioning and superoxide dismutase protect against endothelial dysfunction and endothelium glycocalyx disruption in the postischemic guinea-pig hearts. Mol Cell Biochem 1998, 186:87-97.

27. Cohen MV, Yang XM, Downey JM: Nitric oxide is a preconditioning mimetic and cardioprotectant and is the basis of many available infarct-sparing strategies. Cardiovasc Res 2006, 70:231-239.

28. Jones SP, Bolli R: The ubiquitous role of nitric oxide in cardioprotection. J Mol Cell Cardiol 2006, 40:16-23.

29. Hillegass WB, Dean NA, Liao L, Rhinehart RG, Myers PR: Treatment of no-reflow and impaired flow with the nitric oxide donor nitroprusside following percutaneous coronary interventions: initial human clinical experience. J Am Coll Cardiol 2001, 37:1335-1343.

30. Massoudy P, Zahler S, Freyholdt T, Henze R, Barankay A, Becker $\mathrm{BF}$, Braun SL, Meisner $\mathrm{H}$ : Sodium nitroprusside in patients with compromised left ventricular function undergoing coronary bypass: reduction of cardiac proinflammatory substances. $J$ Thorac Cardiovasc Surg 2000, 119:566-574.

31. Freyholdt T, Massoudy P, Zahler S, Henze R, Barankay A, Becker $\mathrm{BF}$, Meisner $\mathrm{H}$ : Beneficial effect of sodium nitroprusside after coronary artery bypass surgery: pump function correlates inversely with cardiac release of proinflammatory cytokines. $J$ Cardiovasc Pharmacol 2003, 42:372-378.

32. Zucchi R, Limbruno U, Poddighe R, Mariani M, Ronca G: Purine release from isolated rat heart: a new approach to the study of energy metabolism. J Mol Cell Cardiol 1990, 22:815-826.

33. Dauber IM, Lesnefsky EJ, VanBenthuysen KM, Weil JV, Horwitz LD: Reactive oxygen metabolite scavengers decrease functional coronary microvascular injury due to ischemia-reperfusion. Am J Physiol 1991, 260: $\mathrm{H} 42-\mathrm{H} 49$.

34. Becker BF: Towards the physiological function of uric acid. Free Radic Biol Med 1993, 14:615-631.

35. Lundberg JO, Weitzberg E: Extrapulmonary effects of nitric oxide inhalation therapy: time to consider new dosing regimes? Crit Care 2008, 12:406.

36. Chappell D, Jacob M, Hofmann-Kiefer K, Bruegger D, Rehm M, Conzen $P$, Welsch U, Becker BF: Hydrocortisone preserves the vascular barrier by protecting the endothelial glycocalyx. Anesthesiology 2007, 107:776-784.

37. Liu X, Huang Y, Pokreisz P, Vermeersch P, Marsboom G, Swinnen $M$, Verbeken $E$, Santos J, Pellens M, Gillijns $H$, Werf $F$ Van de, Bloch KD, Janssens S: Nitric oxide inhalation improves microvascular flow and decreases infarction size after myocardial ischemia and reperfusion. J Am Coll Cardiol 2007, 50:808-817. 